\title{
PCSK9 inhibition in the management of hyperlipidemia: focus on evolocumab
}

\author{
This article was published in the following Dove Press journal: \\ Vascular Health and Risk Management \\ 9 May 2016 \\ Number of times this article has been viewed
}

\section{Dirk J Blom' \\ Ricardo Dent ${ }^{2}$ \\ Rita C Castro ${ }^{2}$ \\ Peter P Toth ${ }^{3,4}$}

'Division of Lipidology, Department of Medicine, University of Cape Town, Cape Town, South Africa; ${ }^{2}$ Amgen, Inc., Thousand Oaks, CA, ${ }^{3}$ Ciccarone Center for the Prevention of Cardiovascular Disease, Johns Hopkins University School of Medicine, Baltimore, MD, ${ }^{4} \mathrm{CGH}$ Medical Center, Sterling, IL, USA
Correspondence: Dirk J Blom Division of Lipidology, Department of Medicine, Lipid Laboratory, Fifth Floor, Christiaan Barnard Building, UCT Faculty Health Sciences, University of Cape Town, Anzio Road, 7925 Observatory,

Cape Town, South Africa

Email dirk.blom@uct.ac.za
Abstract: Proprotein convertase subtilisin/kexin type 9 (PCSK9) increases low-density lipoprotein cholesterol (LDL-C) concentrations through interference with normal physiologic hepatic LDL receptor (LDLR) recycling. Inhibiting PCSK9 results in improved LDLR recycling, increased LDLR availability on hepatocyte cell surfaces, and reduced blood LDL-C levels, making PCSK9 inhibition a novel therapeutic strategy for managing hypercholesterolemia. Monoclonal antibodies directed against PCSK9 have been developed for this purpose. A large number of clinical trials have demonstrated that monoclonal antibodies against PCSK9 yield substantial reductions in LDL-C when administered as monotherapy or in combination with statins to patients with nonfamilial and familial forms of hypercholesterolemia. Data from long-term trials demonstrate that the LDL-C-lowering effect of PCSK9 inhibitors is durable. These agents are generally well tolerated, and few patients discontinue treatment due to adverse events. Moreover, PCSK9 inhibitors do not appear to elicit the hepatic and muscle-related side effects associated with statin use. The ultimate value of PCSK9 inhibitors will be measured by their effect on clinical outcomes. Early evidence of a reduction in cardiovascular events after 1 year of treatment was shown in a prospective exploratory analysis of two ongoing long-term open-label extension evolocumab trials. Similarly, cardiovascular events were reduced in another exploratory analysis after $>1$ year of therapy with alirocumab. For the primary care physician, PCSK9 inhibitors represent a welcome additional option for lowering LDL-C in patients with familial forms of hypercholesterolemia and those with clinical atherosclerotic cardiovascular disease who are on maximally tolerated statin therapy.

Keywords: alirocumab, monoclonal antibody, hypercholesterolemia, hyperlipidemia, LDL-C

\section{Introduction}

Elevated low-density lipoprotein cholesterol (LDL-C) is a well-established causal risk factor for atherosclerosis. ${ }^{1-4}$ Currently, statins are the mainstay therapy for lowering LDL-C. Statins reduce both cardiovascular events and mortality in patients with elevated risk of atherosclerotic cardiovascular disease. ${ }^{5-8}$ Although statins are the most important LDL-C-lowering drugs, LDL-C lowering beyond that achieved with these agents is desirable and beneficial. The Improved Reduction of Outcomes: Vytorin Efficacy International Trial (IMPROVE-IT) showed that the addition of ezetimibe to simvastatin $40 \mathrm{mg} / \mathrm{d}$ in patients with a recent acute coronary syndrome who had LDL-C levels of 1.3-2.6 mmol/L (50-100 mg/dL) if they were receiving lipid-lowering therapy or $1.3-3.2 \mathrm{mmol} / \mathrm{L}(50-125 \mathrm{mg} / \mathrm{dL})$ if they were not receiving lipid-lowering therapy resulted in additional lowering of LDL-C by an average of $24 \% .{ }^{9}$ This further reduction in LDL-C led to a $13 \%$ relative reduction (absolute: 
$1.7 \%$ reduction) in myocardial infarction $(P=0.002)$ and a $21 \%$ relative reduction (absolute: $0.7 \%$ reduction) in ischemic stroke $(P=0.008)$. These data support evidence from meta-analyses demonstrating merit in attaining very low levels of LDL-C; these meta-analyses demonstrate additional reductions in cardiovascular events in patients achieving LDL-C levels of $<1.8 \mathrm{mmol} / \mathrm{L}(70 \mathrm{mg} / \mathrm{dL})$ or $1.3 \mathrm{mmol} / \mathrm{L}(50 \mathrm{mg} / \mathrm{dL}){ }^{10,11}$ A Cholesterol Treatment Trialists (CTT) meta-analysis demonstrated a 37\% relative risk reduction in major vascular events with LDL-C reductions of $<1.8 \mathrm{mmol} / \mathrm{L}(70 \mathrm{mg} / \mathrm{dL})$ in statin-treated patients. ${ }^{11}$ Post hoc analysis of the Justification for the Use of Statins in Prevention: an Intervention Trial Evaluating Rosuvastatin (JUPITER) trial, which evaluated the efficacy of rosuvastatin as primary prevention in patients with an LDL-C of $<3.4 \mathrm{mmol} / \mathrm{L}(<130 \mathrm{mg} / \mathrm{dL})$ but high-sensitivity C-reactive protein $\geq 2.0 \mathrm{mg} / \mathrm{L}$, found that patients who attained an LDL-C of $<1.3 \mathrm{mmol} / \mathrm{L}(<50 \mathrm{mg} / \mathrm{dL})$ had a $65 \%$ reduction in the occurrence of a cardiovascular event or death from cardiovascular causes $(P<0.0001)$ compared with placebo. By comparison, patients who did not attain an LDL-C of $<1.3 \mathrm{mmol} / \mathrm{L}(<50 \mathrm{mg} / \mathrm{dL})$ achieved only a $24 \%$ reduction. ${ }^{12}$ Likewise, post hoc analysis of the Pravastatin or Atorvastatin Evaluation and Infection Therapy - Thrombolysis in Myocardial Infarction 22 (PROVE IT-TIMI 22) trial, which evaluated the efficacy of moderate (pravastatin, $40 \mathrm{mg} / \mathrm{d}$ ) or intensive (atorvastatin, $80 \mathrm{mg} / \mathrm{d}$ ) statin therapy plus gatifloxacin antibiotic therapy in patients with a recent acute coronary syndrome, found that patients who achieved an LDL-C of 1.0-1.5 mmol/L (40-60 mg/dL) or $<1.0 \mathrm{mmol} / \mathrm{L}(<40 \mathrm{mg} / \mathrm{dL})$ had further reductions in cardiovascular events compared with patients who achieved an LDL-C of $>2.0-2.6 \mathrm{mmol} / \mathrm{L}(>80-100 \mathrm{mg} / \mathrm{dL}) .{ }^{13}$ Post hoc analysis of the Treating to New Targets study showed a significant reduction in the rate of major cardiovascular events across five quintiles of on-treatment-achieved LDL-C concentrations ranging from $\geq 2.7 \mathrm{mmol} / \mathrm{L}(\geq 106 \mathrm{mg} / \mathrm{dL})$ to $<1.6 \mathrm{mmol} / \mathrm{L}(<64 \mathrm{mg} / \mathrm{dL} ; P<0.0001) .{ }^{14}$

Although substantial evidence supports reaching lower LDL-C levels, many statin-treated patients fail to reach the desired LDL-C levels. A meta-analysis of eight statin trials found that out of patients receiving high-dose statin therapy, $13 \%$ failed to reach the LDL-C level of $<2.6 \mathrm{mmol} / \mathrm{L}$ $(<100 \mathrm{mg} / \mathrm{dL})$ and $40 \%$ failed to reach $<1.8 \mathrm{mmol} / \mathrm{L}$ $(<70 \mathrm{mg} / \mathrm{dL}) \cdot{ }^{10}$ Results are more worrisome in community practice with an international survey finding that $70 \%$ of veryhigh-risk patients (of whom $80 \%$ were receiving a statin) failed to reach the LDL-C level of $<1.8 \mathrm{mmol} / \mathrm{L}(<70 \mathrm{mg} / \mathrm{dL}) .{ }^{15}$
Reasons for lack of achieving the desired LDL-C levels are variable and likely include high baseline levels, prescription of inadequate statin doses, poor adherence, and the inability to tolerate an adequate statin dose. Statin-associated muscle symptoms are a frequent cause for patients to not take statins or to only take a low dose. The Prediction of Muscular Risk in Observational conditions (PRIMO) study found that $10 \%$ of patients in community practice who were taking high-dose statins reported muscle symptoms, a rate significantly higher than suggested by placebocontrolled clinical trials. ${ }^{16}$ On average, patients fail to take prescribed statins $\sim 20 \%$ of the time ${ }^{17,18}$ and approximately half of patients discontinue statin therapy by 5 years. ${ }^{17,19}$ Poor adherence with resulting elevated LDL-C levels place patients at risk for hypercholesterolemia-associated morbidity and mortality. Overall, these data demonstrate that additional options are needed to assist patients toward achieving the required LDL-C levels. The recent approval of monoclonal antibodies against proprotein convertase subtilisin/kexin type 9 (PCSK9) by the US Food and Drug Administration and the European Medicines Agency, among other regulatory agencies, provides physicians with another LDL-C-lowering option.

\section{PCSK9}

PCSK9 is a serine protease involved in cholesterol metabolism that is enzymatically inactive following secretion. PCSK9 is a proprotein convertase belonging to the subtilase subfamily. ${ }^{20}$ In healthy humans, plasma PCSK9 levels decrease with fasting and increase following meals. ${ }^{21,22}$ Shortly after the discovery of $P C S K 9,{ }^{23}$ it was shown that a gain-of-function mutation in $P C S K 9$ is associated with familial hypercholesterolemia. ${ }^{20,24}$ Further research demonstrated that loss-of-function mutations in PCSK9 are associated with reduced LDL-C concentrations and that these lifetime reductions confer substantial protection against coronary artery disease. ${ }^{25-27}$ PCSK9 gene expression is regulated by the nuclear transcription factor sterol regulatory elementbinding protein-2. ${ }^{28}$ Levels of sterol regulatory elementbinding protein-2 are increased by statin therapy, which thus also increases PCSK9 levels. PCSK9 inhibition may thus be an especially useful therapeutic strategy in statin-treated patients.

In adults, PCSK9 is expressed predominantly in the liver, and to a lesser extent in the intestine and kidney. ${ }^{23}$ Currently, the only known physiologically relevant function of circulating PCSK9 is to regulate LDL receptor (LDLR) in the liver. PCSK9 increases LDL-C concentrations through interference 
with normal physiologic hepatic LDLR recycling. LDL particles are largely removed from the circulation via the LDLR, which are located on the surface of hepatocytes. The LDLR binds LDL and the complex enters the cell through a clathrin-coated vesicle. Intracellularly, the LDL and LDLR dissociate. LDL is delivered to a lysosome and degraded, while the LDLR is recycled back to the hepatocyte cell surface (Figure 1A). ${ }^{29}$ PCSK9 interferes with this process by preventing the separation of the LDLR from LDL. PCSK9 binds to the cell-surface LDLR; upon LDL binding and internalization, the PCSK9-bound LDLR fails to separate from the LDL particle. As a result, the LDLR is delivered to the lysosome and degraded along with the LDL, thus bypassing the process of recycling to the hepatocyte cell surface (Figure 1B). ${ }^{30}$ The diminished LDLR concentration on hepatocyte cell surfaces results in elevated plasma LDL-C due to reduced clearance of LDL. Inhibiting PCSK9 therefore results in improved LDLR recycling, increased availability of LDLR on hepatocyte cell surfaces, increased LDL plasma clearance, and reduced blood LDL-C levels, making PCSK9 inhibition an effective therapeutic strategy for LDL hypercholesterolemia.

\section{PCSK9 monoclonal antibodies}

Currently, most of the data for PCSK9 inhibition come from studies with monoclonal antibodies that are directed against PCSK9 and prevent its interaction with the LDLR (Table 1 and Figure 1C). ${ }^{31,32}$ Therapeutic monoclonal antibodies play important roles in the management of many inflammatory disorders and cancers because of their ability to bind to a selected target highly specifically, but they have not been widely used in the management of cardiovascular disease as yet. Monoclonal antibodies are target-specific antibodies created through recombinant DNA technology. These proteins have the characteristic Y-shaped protein structure of B-cell-derived antibodies and are designed to bind to a single therapeutic target with high specificity. ${ }^{33}$ Monoclonal antibodies exert their therapeutic action through a variety of mechanisms, including direct effects associated with the binding of the antibody to the target (target blockade, the mechanism by which current anti-PCSK 9 monoclonal antibodies exert their effects) $)^{31,32}$ and indirect effects involving depletion of cells targeted by the monoclonal antibody. ${ }^{33}$ Monoclonal antibodies are administered parenterally (intravenously, intramuscularly, or subcutaneously). ${ }^{34}$ Elimination occurs not through the liver or kidneys, but primarily through antigen-specific target-mediated disposition and nonspecific pathways of the reticuloendothelial system. ${ }^{35}$
Monoclonal antibody structures vary according to the proportion of murine components and production methods. After the development of early murine antibodies, investigators sought to replace murine components with human components to reduce the immunogenicity of the antibodies. ${ }^{36,37}$ Of particular concern is the potential for the development of neutralizing antibodies that can reduce the therapeutic efficacy of monoclonal antibodies. ${ }^{38}$ Further development initially resulted in chimeric antibodies, which consisted of a human antibody with murine variable regions. Next, humanized antibodies were generated, which contained all human components except for the antigen-binding complementarity-determining regions. ${ }^{36}$ Finally, fully human monoclonal antibodies were developed using novel platforms. ${ }^{39,40}$ Evolocumab and alirocumab are fully human anti-PCSK9 monoclonal antibodies, ${ }^{31,41-43}$ while bococizumab is a humanized monoclonal antibody. ${ }^{32}$

\section{Clinical trials}

A large number of clinical trials have now been conducted with anti-PCSK9 antibodies, demonstrating that these agents lead to substantial reductions in LDL-C when administered as monotherapy or in combination with statins and/or ezetimibe to patients with nonfamilial and familial forms of hypercholesterolemia. Published randomized controlled Phase III trials evaluating evolocumab and alirocumab are summarized in Table 2. In patients without homozygous familial hypercholesterolemia (HoFH), evolocumab led to mean reductions in LDL-C of $48 \%-76 \%$ compared with placebo and of $38 \%-47 \%$ compared with ezetimibe after 12 or 52 weeks of treatment. ${ }^{44-48}$ Alirocumab led to mean reductions of $46 \%-62 \%$ compared with placebo and $24 \%-32 \%$ compared with ezetimibe after 24 weeks of treatment. ${ }^{4-55}$ Note that reductions are consistently reported within this review as placebo/control corrected rather than as change from baseline.

\section{Combination with statins}

Adding a PCSK9 inhibitor to background statin therapy offers a useful strategy to further lower LDL-C in patients unable to achieve optimal LDL-C levels with statin therapy. The majority of clinical trials conducted with PCSK9 inhibitors have evaluated this combination and have included a diverse patient population treated with widely varying statin dose intensities. In some trials, additional non-statin lipid-lowering therapy was also allowed with ezetimibe, the most commonly used second agent. ${ }^{44,46,48,49,51-53}$ Mean reductions in LDL-C compared with placebo plus background statin therapy 

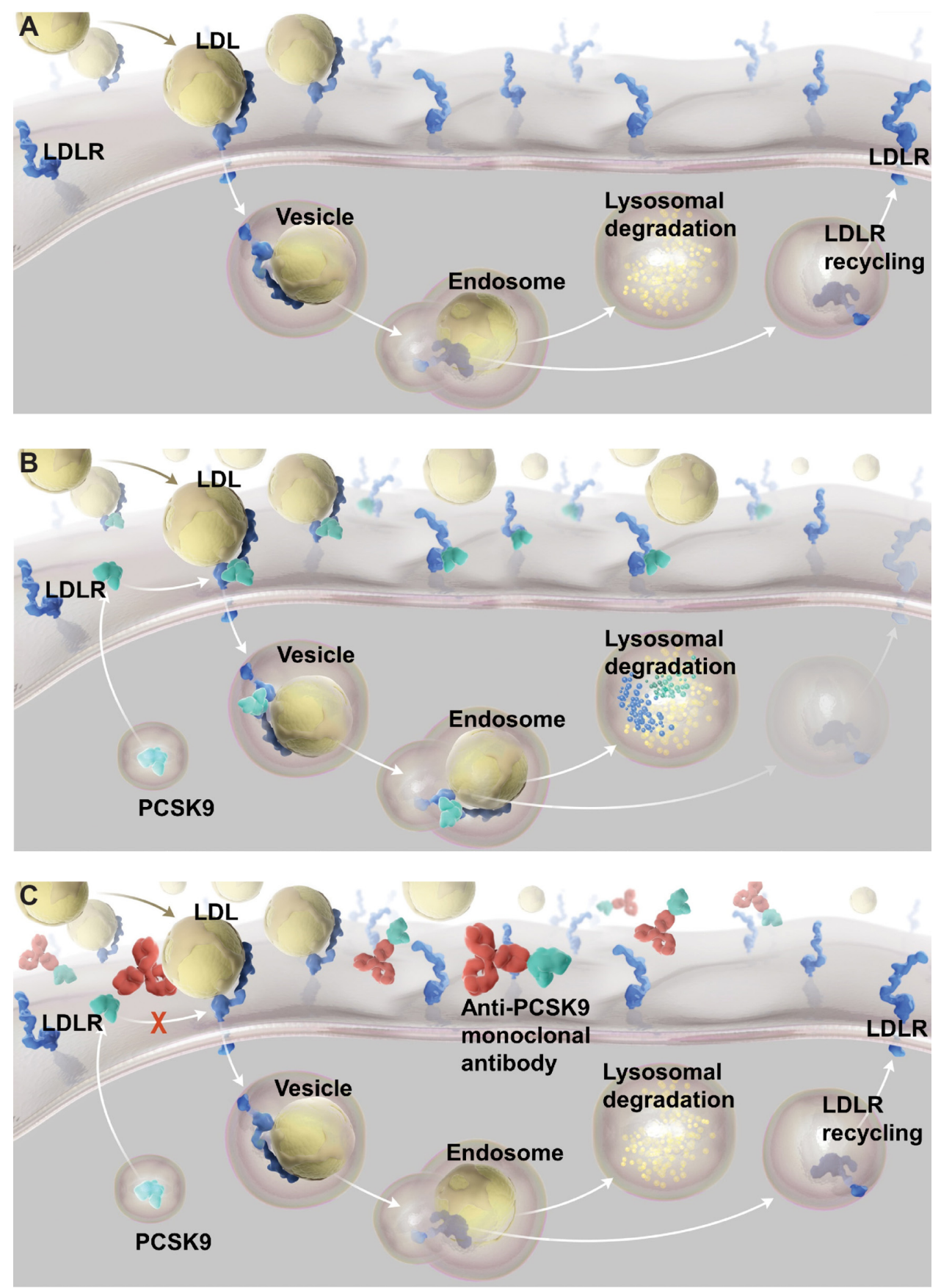

Figure I LDL Recycling, PCSK9 Function, and Effect of PCSK9 Inhibition

Notes: (A) LDLRs are found on the hepatocyte cell surface. Upon binding an LDL particle, the LDLR-LDL particle complex enters the hepatocyte in a clathrin-coated vesicle. Intracellularly, the LDL and LDLR dissociate. LDL is delivered to a lysosome and degraded, while the LDLR is recycled back to the hepatocyte cell surface. (B): PCSK9 interferes with the LDLR recycling by preventing the separation of the LDLR from LDL. PCSK9 binds to the cell-surface LDLR; upon LDL binding and internalization, the PCSK9-bound LDLR fails to separate from the LDL particle. As a result, the LDLR is delivered to the lysosome and degraded along with the LDL, thus bypassing the process of recycling to the hepatocyte cell surface. (C): Monoclonal antibodies directed against PCSK9 prevent its interaction with the LDLR.

Abbreviations: LDL, low-density lipoprotein; LDLRs, LDL receptors; PCSK9, proprotein convertase subtilisin/kexin type 9.

among patients receiving PCSK9 inhibitors in addition to statins, with or without other lipid-lowering therapy, ranged from $48 \%$ to $76 \%$ at week 12 in trials of evolocumab and from $46 \%$ to $62 \%$ at week 24 in trials of alirocumab. ${ }^{44,46,47,51-53}$ Different intensities of baseline therapy do not appear to influence the magnitude of lipid lowering achieved with PCSK9 inhibition. In the Durable Effect of PCSK9 Antibody Compared with Placebo Study (DESCARTES), patients were assigned a statin dose based on their screening LDL-C and National Cholesterol Education Program Adult Treatment 
Table I Anti-PCSK9 monoclonal antibodies marketed or in Phase III development

\begin{tabular}{llll}
\hline Generic name & Brand name & Manufacturer & $\begin{array}{c}\text { Marketing status } \\
\text { dosing regimens }\end{array}$ \\
\hline Evolocumab & Repatha & $\begin{array}{l}\text { Amgen, Inc. (Thousand Oaks, } \\
\text { CA, USA) }\end{array}$ & $\begin{array}{l}\text { Marketed } \\
\text { I40 mg SC Q2Wa } \\
420 \text { mg SC QMa,b }\end{array}$ \\
Alirocumab & Praluent & Sanofi-Aventis and Regeneron & Marketed \\
Bococizumab & - & (Tarrytown, NY, USA) & I50 mg SC Q2W \\
\hline
\end{tabular}

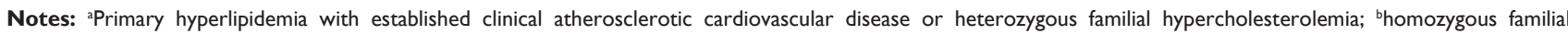
hypercholesterolemia; 'if LDL-C response is inadequate with $75 \mathrm{mg}$ SC Q2W.

Abbreviations: LDL-C, low-density lipoprotein cholesterol; PCSK9, proprotein convertase subtilisin/kexin type 9; Q2W, every 2 weeks; QM, monthly; SC, subcutaneously.

Panel III risk level. Mean LDL-C reductions with monthly evolocumab compared with placebo at week 52 were $48 \%$ in those receiving atorvastatin $80 \mathrm{mg}$ plus ezetimibe $10 \mathrm{mg}$, $57 \%$ in those receiving atorvastatin $80 \mathrm{mg}$ alone, $62 \%$ in those receiving atorvastatin $10 \mathrm{mg}$, and $56 \%$ in those receiving diet alone as their baseline concomitant therapy with evolocumab. ${ }^{44}$ In the LDL-C Assessment with PCSK9 Monoclonal Antibody Inhibition Combined with Statin Therapy-2 (LAPLACE-2) trial, in which patients were randomized to a baseline statin dose, mean LDL-C reductions with every 2-week evolocumab compared with placebo at week 12 were $68 \%-76 \%$ in patients receiving high-intensity stains and $68 \%-71 \%$ in those receiving moderate-intensity statins. ${ }^{47}$

Whether PCSK9 inhibitors can provide additional LDL-C lowering in patients receiving combination therapy with statin and ezetimibe is an important and clinically relevant question in the face of the recently published IMPROVE-IT trial. ${ }^{9}$ In trials that enrolled patients with heterozygous familial hypercholesterolemia $(\mathrm{HeFH})$, more than half of the patients were receiving ezetimibe in addition to their statin. ${ }^{46,51}$ These trials demonstrated robust LDL-C lowering among all patients, and in subgroup analysis of the Reduction of LDL-C with PCSK9 Inhibition in Heterozygous Familial Hypercholesterolemia Disorder (RUTHERFORD-2) trial, evolocumab lowered LDL-C to a similar extent between patients receiving ezetimibe and those who were not. ${ }^{46}$ Additional data are available from the DECARTES trial. Among 189 patients receiving atorvastatin $80 \mathrm{mg}$ plus ezetimibe $10 \mathrm{mg}$ daily, the addition of monthly evolocumab resulted in an average LDL-C reduction of $48 \%$ when compared with placebo. ${ }^{44}$

\section{Monotherapy}

Because statin therapy upregulates PCSK9 expression, it is important to know whether PCSK9 inhibition is less effective in patients who are not receiving statins and thus do not have high PCSK9 levels. The Monoclonal Antibody against PCSK9 to
Reduce Elevated LDL-C in Subjects Currently Not Receiving Drug Therapy for Easing Lipid Levels-2 (MENDEL-2) trial evaluated evolocumab monotherapy in patients with screening LDL-C $\geq 2.6 \mathrm{mmol} / \mathrm{L}(\geq 100 \mathrm{mg} / \mathrm{dL}){ }^{45}$ The LDL-C reduction achieved with evolocumab in this placebo-controlled trial ( $55 \%-57 \%$ vs placebo) was similar to that seen when evolocumab was added to statins. Further evidence comes from the diet-only arm of DESCARTES, which showed that evolocumab monotherapy led to a $56 \%$ LDL-C reduction compared with placebo. ${ }^{44}$ The MENDEL-2 trial also compared evolocumab monotherapy with ezetimibe, finding that evolocumab reduced LDL-C by $38 \%-39 \%$ compared with ezetimibe ${ }^{45}$ Alirocumab was also evaluated as monotherapy in patients with screening LDL-C $\geq 2.6 \mathrm{mmol} / \mathrm{L}$ $(\geq 100 \mathrm{mg} / \mathrm{dL}$ ) in the Efficacy and Safety of Alirocumab SAR236553 (REGN727) vs Ezetimibe in Patients with Hypercholesterolemia (ODYSSEY MONO) trial. This study utilized ezetimibe as its only comparator and alirocumab reduced LDL-C by $32 \%$ vs ezetimibe. ${ }^{54}$ PCSK 9 inhibition is thus an effective lipid-lowering strategy even if LDLRs are not upregulated by statins.

\section{Long-term therapy}

Data from long-term trials demonstrate that the LDL-Clowering effect of PCSK9 inhibitors is durable. Patients who had enrolled in one out of 12 Phase II or III evolocumab trials were eligible to enroll in the Open-label Study of LongTerm Evaluation against LDL Cholesterol (OSLER)-1 and OSLER-2 studies. ${ }^{56}$ In these studies, 4,465 patients were randomized 2:1 to receive evolocumab plus standard therapy or standard therapy alone. At week 12 , evolocumab therapy was associated with a $61 \%$ reduction in LDL-C compared with standard therapy alone, similar to the $58 \%$ reduction observed at 48 weeks. Further long-term data for evolocumab come from the DESCARTES trial, which found a $58 \%$ reduction in LDL-C with monthly evolocumab treatment compared 


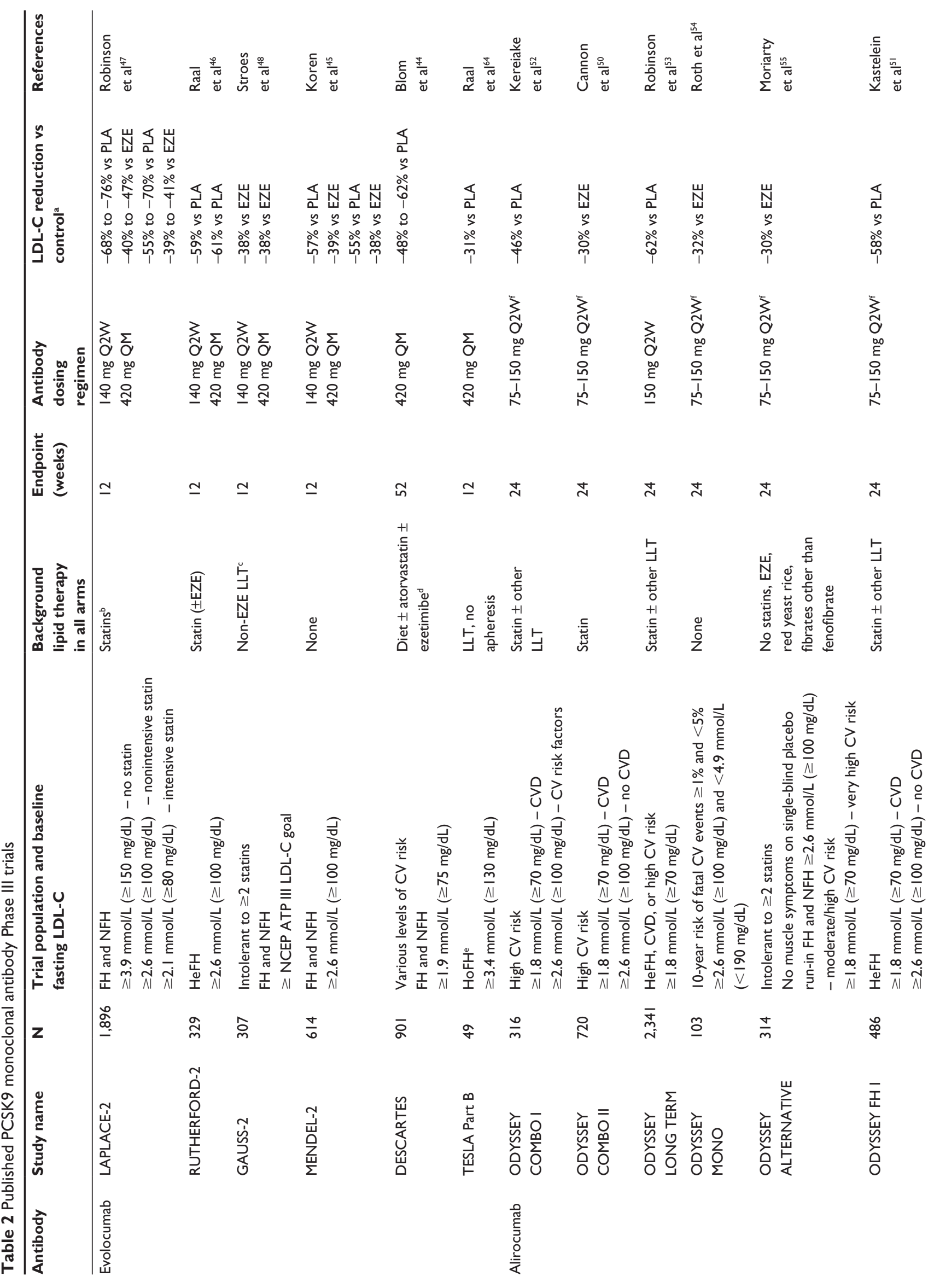




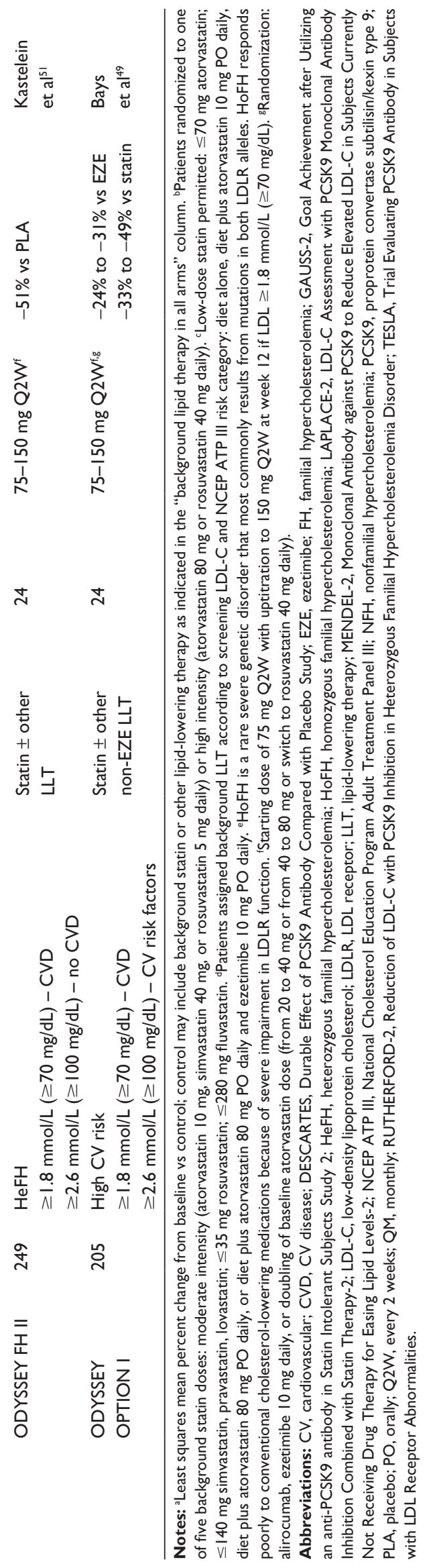

with placebo at week 12 and a $57 \%$ reduction at week $52 .{ }^{44}$ Prolonged therapy with alirocumab was evaluated in the Long-term Safety and Tolerability of Alirocumab in High Cardiovascular Risk Patients with Hypercholesterolemia Not Adequately Controlled with Their Lipid Modifying Therapy (ODYSSEY LONG TERM) trial, which included 2,341 patients who received alirocumab for 78 weeks. ${ }^{53}$ The mean LDL-C reduction at weeks 24 and 78 were $61 \%$ and $52 \%$, respectively, compared with placebo in intention-to-treat analysis. When patients who discontinued therapy early were excluded, the mean reduction at 78 weeks was $58 \%$.

\section{Effect on lipoprotein(a)}

Lipoprotein(a) (Lp(a)) is an independent risk factor for cardiovascular disease ${ }^{57,58}$ and is not lowered by statins or other currently available lipid-lowering therapy apart from nicotinic acid. ${ }^{59}$ PCSK9 inhibition results in statistically significant reductions in plasma $\mathrm{Lp}(\mathrm{a}) .{ }^{45,54}$ When compared with baseline, evolocumab monotherapy reduced Lp(a) by $18 \%-20 \%$ after 12 weeks and alirocumab monotherapy reduced $\mathrm{Lp}$ (a) by $17 \%$ after 24 weeks. ${ }^{45,54}$ When added to moderate- or high-intensity dose statin therapy, reductions in Lp(a) with evolocumab ranged from $24 \%$ to $39 \%$ and the reduction with alirocumab was $28 \%$ in two representative trials. ${ }^{47,50}$ In an analysis of multiple evolocumab trials, reductions in $\mathrm{Lp}$ (a) were positively correlated with LDL-C reductions. ${ }^{60}$ Although the mean percentage reduction in $\mathrm{Lp}$ (a) was significantly greater in those patients with baseline $\mathrm{Lp}(\mathrm{a})$ of $\leq 125 \mathrm{nmol} / \mathrm{L}$, the absolute reduction was substantially larger in those with levels of $>125 \mathrm{nmol} / \mathrm{L}$.

\section{Effect on high-density lipoprotein cholesterol and triglycerides}

Whether PCSK9 inhibitors affect high-density lipoprotein cholesterol (HDL-C) or triglyceride levels is of clinical interest. In the MENDEL-2 trial, evolocumab monotherapy increased HDL-C by $6 \%-9 \%$ compared with placebo $(P<0.01) .{ }^{45}$ Reductions in triglyceride levels were 6\% with evolocumab $140 \mathrm{mg}$ every 2 weeks (Q2W; $P=0.72)$ and $18 \%$ with evolocumab $420 \mathrm{mg}$ every 4 weeks $(P<0.001)$ compared with placebo. In the ODYESSY MONO trial, alirocumab monotherapy increased HDL-C by $6 \%$ compared with ezetimibe $(P=0.02){ }^{54}$ The mean reduction in triglycerides was $2 \%(P=0.74)$ compared with ezetimibe.

\section{Special populations}

\section{Heterozygous familial hypercholesterolemia}

Trials evaluating the addition of PCSK9 inhibitors to maximal therapy with statins plus ezetimibe and/or other lipid-lowering 
therapies have shown substantial mean LDL-C reductions of $51 \%-59 \%$ compared with placebo in patients with HeFH. ${ }^{46,51}$ Data from the RUTHERFORD-2 trial suggest that the nature of the LDLR mutation and its residual function, if a mutation had previously been functionally characterized, does not influence the magnitude of LDL-C lowering in response to PCSK9 inhibition. ${ }^{46}$ In a post hoc exploratory subgroup analysis of patients with a single LDLR causative mutation in this trial, mean reductions for evolocumab vs placebo were $55 \%-61 \%$ for patients with an LDLR-negative mutation, $49 \%-66 \%$ in patients with an LDLR-defective mutation, $62 \%-63 \%$ in patients with an unclassified LDLR mutation, and $43 \%-64 \%$ in patients with no mutation identified. Patients with HeFH have one nonmutated LDLR allele, and upregulation of this fully functional allele likely accounts for the majority of the response, making the contribution, if any, by the mutated allele less relevant.

\section{Homozygous familial hypercholesterolemia}

$\mathrm{HoFH}$ is a rare severe genetic disorder that most commonly results from mutations in both LDLR alleles. LDL-C concentrations are extremely high (typically $>12.9 \mathrm{mmol} / \mathrm{L}$ [500 $\mathrm{mg} / \mathrm{dL}]$ in untreated patients) and premature cardiovascular disease is ubiquitous, often leading to early death. ${ }^{61}$ HoFH responds poorly to conventional cholesterol-lowering medications because of severe impairment in LDLR function, with LDL-C reductions of only $\sim 25 \%$ with statins. ${ }^{62,63}$ Evidence supporting the efficacy of PCSK9 inhibition with evolocumab in patients with HoFH comes from the Trial Evaluating PCSK9 Antibody in Subjects with LDL Receptor Abnormalities (TESLA) Part B trial. ${ }^{64}$ In this study of 49 patients with $\mathrm{HoFH}$ and a baseline mean (standard deviation [SD]) LDL-C of 9.0 (3.5) mmol/L (348 [135] mg/dL) who were receiving statins and other lipid-lowering therapy other than apheresis, the addition of monthly evolocumab led to a mean $31 \%$ reduction in LDL-C compared with placebo. This percentage reduction, while smaller in magnitude than those observed in other populations, corresponded to a mean absolute LDL-C reduction of $2.4 \mathrm{mmol} / \mathrm{L}(94 \mathrm{mg} / \mathrm{dL})$ and is considered clinically meaningful in this high-risk population. Prespecified subgroup analysis of this trial suggested that some residual LDLR functionality is required for LDL-C-lowering activity. Patients with defective LDLR mutation status in at least one allele showed statistically significant LDL-C reductions of $24 \%-47 \%$, while the single patient with negative LDLR mutation status in both alleles experienced a 10\% increase from baseline in LDL-C.

\section{Statin intolerant}

Patients who are unable to tolerate statins due to musclerelated or other side effects represent a population who may benefit from the alternative mechanism of action offered by PCSK9 inhibitors. Due to the subjective nature of muscle symptoms associated with statin therapy and the high prevalence of muscle symptoms in general, regardless of statin treatment, the true number of patients with statin intolerance is difficult to ascertain. ${ }^{65-68}$ The PRIMO study found that $10 \%$ of patients receiving high-dose statins in clinical practice experienced muscle symptoms, which were associated with activity limitations in more than one-third of these patients. ${ }^{16}$ Effective options for LDL-C lowering in patients who cannot tolerate statins are limited. The Goal Achievement after Utilizing an anti-PCSK9 antibody in Statin Intolerant Subjects Study 2 (GAUSS-2) trial evaluated evolocumab in 307 patients who had failed two or more statins and could tolerate either no statin or only a low dose of statin. ${ }^{48}$ The majority of patients had discontinued prior statins due to myalgia. However, 20\% of patients had experienced either myositis (defined as muscle symptoms with creatine kinase elevation) or rhabdomyolysis. Notably, 56\% of patients were at high risk for coronary heart disease as classified by the National Cholesterol Education Program. Patients were randomized to receive either ezetimibe or evolocumab (either $140 \mathrm{mg}$ Q2W or $420 \mathrm{mg}$ monthly). Both regimens of evolocumab resulted in a mean $38 \%$ reduction in LDL-C compared with ezetimibe. Evolocumab was well tolerated in this population of patients who had a strong history of muscle symptoms with statin use. Myalgia was reported in 7\%-9\% of patients receiving evolocumab and $18 \%$ of patients receiving ezetimibe. Data from the ODYSSEY ALTERNATIVE trial indicate that alirocumab is also effective in a population of patients who had failed two statins for muscle symptoms. ${ }^{55}$ This trial included a single-blind placebo run-in phase $(\mathrm{N}=361)$ during which 47 patients who experienced muscle symptoms (13\%) were excluded. Additionally, an atorvastatin $20 \mathrm{mg}$ control arm was included along with an ezetimibe control, and all arms were placebo controlled. Results from this trial demonstrated a $30 \%$ reduction in LDL-C with alirocumab compared with ezetimibe. Interestingly, musclerelated adverse events were statistically significantly lower in the alirocumab arm compared with the atorvastatin arm ( $32 \%$ vs $46 \% ; P=0.042$ ). While demonstrating the efficacy of PCSK9 inhibition in this stain-intolerant population, the ODYSSEY ALTERNATIVE trial also adds to the body of literature illustrating the complexity and multifaceted nature of statin-associated muscle symptoms with a significant 
proportion of patients excluded during the placebo run-in while many patients were able to take atorvastatin.

\section{Safety}

Evolocumab is generally well tolerated and few patients discontinued this agent due to adverse events. In the placebocontrolled evolocumab monotherapy trial, MENDEL-2, adverse event rates were comparable between evolocumab and placebo (44\% in each arm).$^{45}$ Serious events occurred in $1.3 \%$ of patients receiving evolocumab and $0.6 \%$ of patients on placebo. Adverse events led to study drug discontinuation in $2.3 \%$ of patients receiving evolocumab and $3.9 \%$ of patients receiving placebo. Injection-site reactions occurred in $5 \%$ of each arm. The alirocumab monotherapy trial, ODYSSEY MONO, was ezetimibe controlled; adverse events occurred in $69 \%$ of patients receiving alirocumab and $78 \%$ of patients receiving ezetimibe. ${ }^{54}$ Statin combination trials revealed no increase in adverse events when PCSK9 inhibitors were added to statins. ${ }^{47,52}$ Longer term studies showed continued tolerability. After 52 weeks of evolocumab treatment, $2 \%$ of patients discontinued treatment for an adverse event compared with $1 \%$ of patients receiving placebo. ${ }^{44}$ After 78 weeks of alirocumab treatment, $7 \%$ of patients discontinued for an adverse event compared with $6 \%$ of patients receiving placebo. ${ }^{53}$ The most common adverse events occurring in clinical trials of evolocumab and alirocumab are summarized in Table 3.42,43

A potential concern with long-term administration of biologic agents is the development of neutralizing antidrug antibodies and loss of efficacy over time. Pooled data from placebo- and active-controlled evolocumab trials demonstrate that $0.1 \%$ of evolocumab-treated patients developed binding antidrug antibodies with no neutralizing antibody formation; these binding antibodies were not associated with altered pharmacokinetics, safety, or clinical response. ${ }^{43}$ Pooled data from ten placebo- and active-controlled alirocumab trials demonstrated binding antidrug antibodies in $4.8 \%$ of alirocumab-treated patients and $0.6 \%$ of control-treated patients. ${ }^{42}$ These patients experienced a higher rate of injection-site reactions compared with those without antidrug antibodies (10.2\% vs 5.9\%). Neutralizing antibodies developed in $1.2 \%$ of alirocumab-treated patients and no control-treated patients; $0.3 \%$ of patients had both neutralizing antibodies and transient or prolonged loss of efficacy.

Diabetes and neurocognitive events are of potential concern with statins. ${ }^{70-72}$ Whether these same side effects occur with PCSK9 inhibition is of interest and has been evaluated in trials. In DESCARTES, mean (standard error) fasting
Table 3 Adverse events occurring in $\geq 3 \%$ of monoclonal antibodytreated patients and more frequently than with placebo

\begin{tabular}{|c|c|c|}
\hline Adverse event & Placebo (\%) & $\begin{array}{l}\text { Monoclonal } \\
\text { antibody }^{\mathrm{a}}(\%)\end{array}$ \\
\hline $\begin{array}{l}\text { Evolocumab - 52-week trial } \\
\text { (DESCARTES) }\end{array}$ & $\mathrm{N}=302$ & $\mathrm{~N}=599$ \\
\hline Nasopharyngitis & 9.6 & 10.5 \\
\hline Upper respiratory tract infection & 6.3 & 9.3 \\
\hline Influenza & 6.3 & 7.5 \\
\hline Back pain & 5.6 & 6.2 \\
\hline Injection-site reactions ${ }^{b}$ & 5.0 & 5.7 \\
\hline Cough & 3.6 & 4.5 \\
\hline Urinary tract infection & 3.6 & 4.5 \\
\hline Sinusitis & 3.0 & 4.2 \\
\hline Headache & 3.6 & 4.0 \\
\hline Myalgia & 3.0 & 4.0 \\
\hline Dizziness & 2.6 & 3.7 \\
\hline Musculoskeletal pain & 3.0 & 3.3 \\
\hline Hypertension & 2.3 & 3.2 \\
\hline Diarrhea & 2.6 & 3.0 \\
\hline Gastroenteritis & 2.0 & 3.0 \\
\hline Evolocumab - seven pooled & $N=1,224$ & $N=2,052$ \\
\hline \multicolumn{3}{|c|}{ I 2-week placebo-controlled trials } \\
\hline Nasopharyngitis & 3.9 & 4.0 \\
\hline \multicolumn{3}{|l|}{ placebo-controlled trials ${ }^{c}$} \\
\hline Nasopharyngitis & $\mathrm{II} . \mathrm{I}$ & 11.3 \\
\hline Injection-site reactions ${ }^{d}$ & 5.1 & 7.2 \\
\hline Influenza & 4.6 & 5.7 \\
\hline Urinary tract infections & 4.6 & 4.8 \\
\hline Diarrhea & 4.4 & 4.7 \\
\hline Bronchitis & 3.8 & 4.3 \\
\hline Myalgia & 3.4 & 4.2 \\
\hline Muscle spasms & 2.4 & 3.1 \\
\hline Sinusitis & 2.7 & 3.0 \\
\hline
\end{tabular}

Notes: ${ }^{a}$ Both indicated doses combined; 'berythema, pain, and bruising; 'median treatment duration, 65 weeks; derythema/redness, itching, swelling, pain/tenderness. Data from references 42 and 43.

Abbreviation: DESCARTES, Durable Effect of PCSK9 Antibody Compared with Placebo Study.

glucose increased by $0.07(0.04) \mathrm{mmol} / \mathrm{L}(1.3[0.7] \mathrm{mg} / \mathrm{dL})$ from baseline with evolocumab compared with $0.02(0.05)$ $\mathrm{mmol} / \mathrm{L}(0.4[0.9] \mathrm{mg} / \mathrm{dL})$ with placebo. The mean (standard error) glycated hemoglobin increase was $0.02 \%(0.02)$ with evolocumab vs $0 \%$ (0.03) with placebo. ${ }^{44}$ In the ODYSSEY LONG TERM trial, with $>78$ weeks of treatment, new onset diabetes occurred in 1.8\% (alirocumab) and 2.0\% (placebo) of patients and worsening of diabetes occurred in $12.9 \%$ (alirocumab) and $13.6 \%$ (placebo) of patients. ${ }^{53}$ Whether statins are associated with memory loss or other adverse effects on cognition remains controversial. ${ }^{73}$ Any purported mechanism by which memory loss or neurocognitive deficits could occur remains clinically poorly characterized and mechanistically inadequately studied and defined. Preclinical research suggests statins may be associated with a morphologic change 
in neurons consisting of areas of swelling referred to as "beads on a string". ${ }^{74}$ However, whether these changes result from a direct effect of the statin molecule, its mechanism of action, cholesterol lowering (considering that the majority of cholesterol in the brain is derived from local synthesis), ${ }^{75}$ or another cause is unknown. In clinical trials of PCSK9 monoclonal antibodies, neurocognitive events were infrequent. In the 1-year controlled period of the OSLER-1 and OSLER-2 trials, neurocognitive events occurred in $0.9 \%$ and $0.3 \%$ of patients receiving evolocumab plus standard therapy and patients receiving standard therapy alone, respectively. ${ }^{56}$ A dedicated study of cognition (Evaluating PCSK9 Binding antiBody Influence oN coGnitive HeAlth in High cardiovascUlar Risk Subjects [EBBINGHAUS]; NCT02207634), which has enrolled subjects participating in the larger ongoing Further Cardiovascular Outcomes Research with PCSK9 Inhibition in Subjects with Elevated Risk (FOURIER) evolocumab cardiovascular outcomes trial (NCT01764633), is fully enrolled and will provide more detailed, prospectively collected information on the effects of evolocumab on neurocognitive function. In the ODYSSEY LONG TERM trial, neurocognitive disorders occurred in $1.2 \%$ and $0.5 \%$ of patients with alirocumab and placebo, respectively. ${ }^{53}$

The magnitude of LDL-C lowering afforded by PCSK9 inhibition, particularly when added to existing lipid-lowering therapy, raises concerns about hypothetical risks associated with the attainment of very low LDL-C concentrations, including hemorrhagic stroke, vitamin E deficiency, and impaired steroid hormone synthesis. An analysis of 1,104 patients who entered the long-term extension trial (OSLER-1) after participation in Phase II studies found no increase in overall adverse events, serious adverse events, creatine kinase elevations, or liver enzyme elevations in patients who achieved LDL-C concentrations of $<0.6 \mathrm{mmol} / \mathrm{L}(<25 \mathrm{mg} / \mathrm{dL})$ or $<1.3 \mathrm{mmol} / \mathrm{L}(<50 \mathrm{mg} / \mathrm{dL})$ compared with those who experienced LDL-C $\geq 1.3 \mathrm{mmol} / \mathrm{L}$ ( $\geq 50 \mathrm{mg} / \mathrm{dL}) .{ }^{69}$ A numerical increase in headache, insomnia, dizziness, and back pain occurred in patients with lower LDL-C levels. Preliminary data from an analysis of 2,836 patients enrolled in two evolocumab trials, DESCARTES and OSLER-1, demonstrated no increase in rates of adverse events and no cases of hemorrhagic stroke among patients who experienced levels of LDL-C $<1.0 \mathrm{mmol} / \mathrm{L}(<40 \mathrm{mg} / \mathrm{dL}$; $\mathrm{n}=1005)$ or $<0.6 \mathrm{mmol} / \mathrm{L}(<25 \mathrm{mg} / \mathrm{dL} ; \mathrm{n}=644) .{ }^{76}$ Because lipoproteins are involved in vitamin $\mathrm{E}$ transport ${ }^{77}$ and cholesterol is required for steroidogenesis, ${ }^{78}$ levels of these compounds in patients who experienced very low LDL-C levels are of interest. Data from the DESCARTES study were analyzed to answer this question. ${ }^{79,80}$ In all patients receiving evolocumab, absolute vitamin E levels decreased by a mean of $16 \%$; however, cholesterol-normalized vitamin $E$ levels increased by a mean of $19 \%$. The pattern of vitamin $\mathrm{E}$ changes were consistent between patients with very low LDL-C concentrations (defined as $<0.4 \mathrm{mmol} / \mathrm{L}[<15 \mathrm{mg} / \mathrm{dL}],<0.6 \mathrm{mmol} / \mathrm{L}$ [ $<25 \mathrm{mg} / \mathrm{dL}]$, and $<1.0 \mathrm{mmol} / \mathrm{L}[<40 \mathrm{mg} / \mathrm{dL}]$ ) and higher LDL-C concentrations $(\geq 1.0 \mathrm{mmol} / \mathrm{L}[\geq 40 \mathrm{mg} / \mathrm{dL}])$. An analysis of steroid hormones found no correlation between changes in cortisol, testosterone, or estradiol and the change in LDL-C from baseline among evolocumab-treated patients. Overall, these data suggest that the attainment of very low LDL-C concentrations with PCSK9 inhibition poses little risk to patients.

\section{Cardiovascular outcomes}

The ultimate value of PCSK9 inhibitors will be measured by their effect on clinical outcomes. Early evidence of cardiovascular benefit with evolocumab was shown in a prospective exploratory analysis of the OSLER-1 and OSLER-2 studies. ${ }^{56}$ Similarly, post hoc analysis was conducted with alirocumab in the ODYSSEY LONG TERM trial. ${ }^{53}$ Both analyses showed a reduction in major cardiovascular events with evolocumab and alirocumab added to standard therapy compared with standard therapy alone. These data should be interpreted with caution because event numbers are low, but they are reassuring and corroborate other trials demonstrating that additional lowering of LDL-C beyond that achieved with traditional medications results in improved cardiovascular outcomes. ${ }^{9-12}$ Large cardiovascular outcome trials are underway with evolocumab (FOURIER; NCT01764633), alirocumab (ODYSSEY OUTCOMES; NCT01663402), and bococizumab (SPIRE-1; NCT01975376 and SPIRE-2; NCT01975389).

\section{Primary care practice considerations}

For the primary care physician, PCSK9 inhibitors represent a valuable and efficacious addition to the treatment armamentarium for lowering LDL-C in patients who require further lipid lowering beyond statins or are unable to tolerate statins. The mechanism by which these agents lower LDL-C is well defined and supported by results observed in patients who harbor loss-of-function mutations in PCSK9. ${ }^{25,26}$ PCSK9 inhibitors lower LDL-C from $46 \%$ to $76 \%$ compared with placebo, ${ }^{44,46,47,51-53}$ whereas ezetimibe produces an approximate $16 \%-18 \%$ further reduction, ${ }^{49}$ making these monoclonal antibodies attractive for patients who require substantial additional LDL-C lowering beyond statin therapy. The use of these agents is warranted in patients with familial 
forms of hypercholesterolemia, and in patients with clinical atherosclerotic cardiovascular disease on maximally tolerated statins who require additional lowering of LDL-C.

Practical considerations play a role in patient acceptance and adherence to these agents. Evolocumab can be dosed Q2W or monthly. These prolonged dosing intervals lessen the burden on patients and may promote adherence. Data with evolocumab demonstrate that the two dosing frequencies (140 mg Q2W and $420 \mathrm{mg}$ monthly) are clinically equivalent. ${ }^{45-48}$ Thus far, published alirocumab trials have utilized every 2 -week dosing. Results with monthly dosing are available in preliminary form (NCT01926782, NCT02023879) and suggest that monthly dosing of alirocumab may also be possible. ${ }^{81,82}$ However, monthly dosing is currently only approved for evolocumab. ${ }^{42,43}$

PCSK9 monoclonal antibodies are self-administered subcutaneously, and willingness to self-inject or the availability of a caregiver for injections is necessary. Approximately three-quarters $(74.1 \%)$ of eligible patients from evolocumab studies decided to enroll in the OSLER extension studies, with the majority of the remaining $25.9 \%$ of subjects who chose not to participate citing either personal reasons or the level of commitment required, indicating good patient acceptance of an injectable therapy. ${ }^{56}$

These agents are expensive when compared with statins, and funding constraints may limit the number of patients who have access. Evolocumab and alirocumab should be prescribed to carefully selected patients (ie, those patients in whom the absolute risk reduction is largest with further LDL-C reduction) with adequate follow-up to ensure adherence. At present, no comparative data are available to guide clinicians in the choice between anti-PCSK9 monoclonal antibodies.

\section{Conclusion}

PCSK9 inhibition with monoclonal antibodies is a novel therapeutic approach for lowering LDL-C. These agents offer substantial LDL-C lowering in patients with familial forms of hypercholesterolemia and those with clinical atherosclerotic cardiovascular disease who are on maximally tolerated statins. Evolocumab and alirocumab are generally well tolerated and the prolonged (Q2W and monthly) dosing schedules may offer the benefit of high patient adherence. Results from cardiovascular outcome trials are eagerly awaited.

\section{Acknowledgments}

The authors thank Meera Kodukulla, PhD, CMPP of Amgen, Inc. (Thousand Oaks, CA, USA) and Laura Evans, PharmD, on behalf of Amgen Inc., for preliminary drafting of the manuscript.

\section{Disclosure}

Dr Dirk J Blom has received research grants and clinical trial payments from Amgen, Inc. (Thousand Oaks, CA, USA) and Sanofi-Aventis (Bridgewater, NJ, USA), and speaker's honoraria from Amgen, Inc. Dr Dirk J Blom has served as an advisory board member for Amgen, Inc. and Sanofi-Aventis. Dr Peter P Toth is a member of the speaker's bureau for Amarin Corporation, (Dublin, Ireland), Kowa Pharmaceuticals America, Inc. (Montgomery, AL, USA), Merck \& Co., Inc. (Whitehouse Station, NJ, USA), Sanofi-Aventis, and Novartis International AG (Basel, Switzerland), and a consultant for Amgen, Inc., Kowa Pharmaceuticals America, Inc., Merck \& Co., Inc., Sanofi-Aventis, and Novartis International AG. Drs Ricardo Dent and Rita Castro are employees and stockholders of Amgen, Inc. The authors report no other conflicts of interest in this work.

\section{References}

1. The lipid research clinics coronary primary prevention trial results. I. Reduction in incidence of coronary heart disease. JAMA. 1984;251(3):351-364.

2. The lipid research clinics coronary primary prevention trial results. II. The relationship of reduction in incidence of coronary heart disease to cholesterol lowering. JAMA. 1984;251(3):365-374.

3. Stamler J, Wentworth D, Neaton JD. Is relationship between serum cholesterol and risk of premature death from coronary heart disease continuous and graded? Findings in 356,222 primary screenees of the multiple risk factor intervention trial (MRFIT). JAMA. 1986;256(20):2823-2828.

4. Wilson PW, D'Agostino RB, Levy D, Belanger AM, Silbershatz H, Kannel WB. Prediction of coronary heart disease using risk factor categories. Circulation. 1998;97(18):1837-1847.

5. Anderson TJ, Gregoire J, Hegele RA, et al. 2012 update of the Canadian Cardiovascular Society guidelines for the diagnosis and treatment of dyslipidemia for the prevention of cardiovascular disease in the adult. Can J Cardiol. 2013;29(2):151-167.

6. Expert Dyslipidemia P, Grundy SM. An International Atherosclerosis Society position paper: global recommendations for the management of dyslipidemia. J Clin Lipidol. 2013;7(6):561-565.

7. Perk J, De Backer G, Gohlke H, et al. European guidelines on cardiovascular disease prevention in clinical practice (version 2012). The fifth joint task force of the European Society of Cardiology and other societies on cardiovascular disease prevention in clinical practice (constituted by representatives of nine societies and by invited experts). Eur Heart $J$. 2012;33(13):1635-1701

8. Stone NJ, Robinson JG, Lichtenstein AH, et al. 2013 ACC/AHA guideline on the treatment of blood cholesterol to reduce atherosclerotic cardiovascular risk in adults: a report of the American College of Cardiology/American Heart Association Task Force on Practice Guidelines. Circulation. 2014;129(25 suppl 2):S1-S45.

9. Canon CP, Blazing MA, Giugliano RP, et al. Ezetimibe added to statin therapy after acute coronary syndromes. $N$ Engl $\mathrm{J} \mathrm{Med}$. 2015;372(25):2387-2397.

10. Boekholdt SM, Hovingh GK, Mora S, et al. Very low levels of atherogenic lipoproteins and the risk for cardiovascular events: a meta-analysis of statin trials. J Am Coll Cardiol. 2014;64(5):485-494. 
11. Cholesterol Treatment Trialists' (CTT) Collaboration; Baigent C, Blackwell L, Emberson J, et al. Efficacy and safety of more intensive lowering of LDL cholesterol: a meta-analysis of data from 170,000 participants in 26 randomised trials. Lancet. 2010;376(9753):1670-1681.

12. Hsia J, MacFadyen JG, Monyak J, Ridker PM. Cardiovascular event reduction and adverse events among subjects attaining low-density lipoprotein cholesterol $<50 \mathrm{mg} / \mathrm{dl}$ with rosuvastatin. The JUPITER trial (justification for the use of statins in prevention: an intervention trial evaluating rosuvastatin). J Am Coll Cardiol. 2011;57(16):1666-1675.

13. Wiviott SD, Cannon CP, Morrow DA, et al. Can low-density lipoprotein be too low? The safety and efficacy of achieving very low low-density lipoprotein with intensive statin therapy: a PROVE IT-TIMI 22 substudy. J Am Coll Cardiol. 2005;46(8):1411-1416.

14. LaRosa JC, Grundy SM, Kastelein JJ, et al; Treating to New Targets (TNT) Steering Committee and Investigators. Steering Committee and Investigators,. Safety and efficacy of atorvastatin-induced very lowdensity lipoprotein cholesterol levels in patients with coronary heart disease (a post hoc analysis of the treating to new targets [TNT] study). Am J Cardiol. 2007;100(5):747-752.

15. Waters DD, Brotons C, Chiang CW, et al. Lipid treatment assessment project 2: a multinational survey to evaluate the proportion of patients achieving low-density lipoprotein cholesterol goals. Circulation. 2009;120(1):28-34.

16. Bruckert E, Hayem G, Dejager S, Yau C, Begaud B. Mild to moderate muscular symptoms with high-dosage statin therapy in hyperlipidemic patients - the PRIMO study. Cardiovasc Drugs Ther. 2005;19(6):403-414.

17. Ellis JJ, Erickson SR, Stevenson JG, Bernstein SJ, Stiles RA, Fendrick AM. Suboptimal statin adherence and discontinuation in primary and secondary prevention populations. J Gen Intern Med. 2004;19(6):638-645.

18. Erickson SR, Lin YN. Geospatial analysis of statin adherence using pharmacy claims data in the state of Michigan. J Manag Care Spec Pharm. 2014;20(12):1208-1215.

19. Avorn J, Monette J, Lacour A, et al. Persistence of use of lipid-lowering medications: a cross-national study. JAMA. 1998;279(18):1458-1462.

20. Abifadel M, Varret M, Rabes JP, et al. Mutations in PCSK9 cause autosomal dominant hypercholesterolemia. Nat Genet. 2003;34(2):154-156.

21. Browning JD, Horton JD. Fasting reduces plasma proprotein convertase, subtilisin/kexin type 9 and cholesterol biosynthesis in humans. J Lipid Res. 2010;51(11):3359-3363.

22. Persson L, Cao G, Stahle L, et al. Circulating proprotein convertase subtilisin kexin type 9 has a diurnal rhythm synchronous with cholesterol synthesis and is reduced by fasting in humans. Arterioscler Thromb Vasc Biol. 2010;30(12):2666-2672.

23. Seidah NG, Benjannet $\mathrm{S}$, Wickham L, et al. The secretory proprotein convertase neural apoptosis-regulated convertase 1 (NARC-1): liver regeneration and neuronal differentiation. Proc Natl Acad Sci U S A. 2003;100(3):928-933.

24. Abifadel M, Rabes JP, Devillers M, et al. Mutations and polymorphisms in the proprotein convertase subtilisin kexin 9 (PCSK9) gene in cholesterol metabolism and disease. Hum Mutat. 2009;30(4):520-529.

25. Cohen J, Pertsemlidis A, Kotowski IK, Graham R, Garcia CK, Hobbs HH. Low LDL cholesterol in individuals of African descent resulting from frequent nonsense mutations in PCSK9. Nat Genet. 2005;37(2):161-165.

26. Cohen JC, Boerwinkle E, Mosley TH Jr, Hobbs HH. Sequence variations in PCSK9, low LDL, and protection against coronary heart disease. N Engl J Med. 2006;354(12):1264-1272.

27. Lopez D. PCSK9: an enigmatic protease. Biochim Biophys Acta. 2008;1781(4):184-191.

28. Dubuc G, Chamberland A, Wassef H, et al. Statins upregulate PCSK9, the gene encoding the proprotein convertase neural apoptosis-regulated convertase-1 implicated in familial hypercholesterolemia. Arterioscler Thromb Vasc Biol. 2004;24(8):1454-1459.

29. Dunn KW, McGraw TE, Maxfield FR. Iterative fractionation of recycling receptors from lysosomally destined ligands in an early sorting endosome. J Cell Biol. 1989;109(6 pt 2):3303-3314.
30. Zhang DW, Lagace TA, Garuti R, et al. Binding of proprotein convertase subtilisin/kexin type 9 to epidermal growth factor-like repeat A of low density lipoprotein receptor decreases receptor recycling and increases degradation. J Biol Chem. 2007;282(25):18602-18612.

31. Chan JC, Piper DE, Cao Q, et al. A proprotein convertase subtilisin/ kexin type 9 neutralizing antibody reduces serum cholesterol in mice and nonhuman primates. Proc Natl Acad Sci U S A. 2009;106(24): 9820-9825.

32. Liang H, Chaparro-Riggers J, Strop P, et al. Proprotein convertase substilisin/kexin type 9 antagonism reduces low-density lipoprotein cholesterol in statin-treated hypercholesterolemic nonhuman primates. J Pharmacol Exp Ther. 2012;340(2):228-236.

33. Murphy K, Travers P, Walport M. Janeway's Immunobiology. Seventh ed. New York, NY: Garland Science, Taylor \& Francis Group; 2008.

34. Lobo ED, Hansen RJ, Balthasar JP. Antibody pharmacokinetics and pharmacodynamics. J Pharm Sci. 2004;93(11):2645-2668.

35. Tabrizi MA, Tseng CM, Roskos LK. Elimination mechanisms of therapeutic monoclonal antibodies. Drug Discov Today. 2006;11(1-2): $81-88$.

36. Weiner LM. Fully human therapeutic monoclonal antibodies. J Immunother. 2006;29(1):1-9.

37. Yang XD, Jia XC, Corvalan JR, Wang P, Davis CG. Development of ABX-EGF, a fully human anti-EGF receptor monoclonal antibody, for cancer therapy. Crit Rev Oncol Hematol. 2001;38(1):17-23.

38. Hwang WY, Foote J. Immunogenicity of engineered antibodies. Methods. 2005;36(1):3-10.

39. Lonberg N. Human antibodies from transgenic animals. Nat Biotechnol. 2005;23(9):1117-1125.

40. Lonberg N. Fully human antibodies from transgenic mouse and phage display platforms. Curr Opin Immunol. 2008;20(4):450-459.

41. McKenney JM, Koren MJ, Kereiakes DJ, Hanotin C, Ferrand AC, Stein EA. Safety and efficacy of a monoclonal antibody to proprotein convertase subtilisin/kexin type 9 serine protease, SAR236553/ REGN727, in patients with primary hypercholesterolemia receiving ongoing stable atorvastatin therapy. J Am Coll Cardiol. 2012;59(25): 2344-2353.

42. PRALUENT (alirocumab) [package insert]. Bridgewater, NJ: Sanofi-Aventis U.S. LLC; 2015.

43. REPATHA (evolocumab) [package insert]. Thousand Oaks, CA: Amgen, Inc; 2015

44. Blom DJ, Hala T, Bolognese M, et al. A 52-week placebo-controlled trial of evolocumab in hyperlipidemia. $N$ Engl J Med. 2014;370(19): 1809-1819.

45. Koren MJ, Lundqvist P, Bolognese M, et al. Anti-PCSK9 monotherapy for hypercholesterolemia: the MENDEL-2 randomized, controlled phase III clinical trial of evolocumab. $J$ Am Coll Cardiol. 2014;63(23):2531-2540.

46. Raal FJ, Stein EA, Dufour R, et al. PCSK9 inhibition with evolocumab (AMG 145) in heterozygous familial hypercholesterolaemia (RUTHERFORD-2): a randomised, double-blind, placebo-controlled trial. Lancet. 2015;385(9965):331-340.

47. Robinson JG, Nedergaard BS, Rogers WJ, et al. Effect of evolocumab or ezetimibe added to moderate- or high-intensity statin therapy on LDL-C lowering in patients with hypercholesterolemia: the LAPLACE-2 randomized clinical trial. JAMA. 2014;311(18):1870-1882.

48. Stroes E, Colquhoun D, Sullivan D, et al. Anti-PCSK9 antibody effectively lowers cholesterol in patients with statin intolerance: the GAUSS-2 randomized, placebo-controlled phase 3 clinical trial of evolocumab. J Am Coll Cardiol. 2014;63(23):2541-2548.

49. Bays H, Gaudet D, Weiss R, et al. Alirocumab as add-on to atorvastatin versus other lipid treatment strategies: ODYSSEY OPTIONS I randomized trial. J Clin Endocrinol Metab. 2015;100(8):3140-3148.

50. Cannon CP, Cariou B, Blom D, et al. Efficacy and safety of alirocumab in high cardiovascular risk patients with inadequately controlled hypercholesterolaemia on maximally tolerated doses of statins: the ODYSSEY COMBO II randomized controlled trial. Eur Heart $J$. 2015;36(19):1186-1194. 
51. Kastelein JJ, Ginsberg HN, Langslet G, et al. ODYSSEY FH I and FH II: 78 week results with alirocumab treatment in 735 patients with heterozygous familial hypercholesterolaemia. Eur Heart J. 2015;36(43): 2996-3003.

52. Kereiakes DJ, Robinson JG, Cannon CP, et al. Efficacy and safety of the proprotein convertase subtilisin/kexin type 9 inhibitor alirocumab among high cardiovascular risk patients on maximally tolerated statin therapy: the ODYSSEY COMBO I study. Am Heart J. 2015;169(6):906-915e913.

53. Robinson JG, Farnier M, Krempf M, et al. Efficacy and safety of alirocumab in reducing lipids and cardiovascular events. $N$ Engl J Med. 2015;372(16):1489-1499.

54. Roth EM, Taskinen MR, Ginsberg HN, et al. Monotherapy with the PCSK9 inhibitor alirocumab versus ezetimibe in patients with hypercholesterolemia: results of a 24 week, double-blind, randomized phase 3 trial. Int J Cardiol. 2014;176(1):55-61.

55. Moriarty PM, Thompson PD, Cannon CP, et al. Efficacy and safety of alirocumab vs ezetimibe in statin-intolerant patients, with a statin rechallenge arm: the ODYSSEY ALTERNATIVE randomized trial. J Clin Lipidol. 2015;9(6):758-769.

56. Sabatine MS, Giugliano RP, Wiviott SD, et al. Efficacy and safety of evolocumab in reducing lipids and cardiovascular events. $N$ Engl $\mathrm{J} \mathrm{Med}$. 2015;372(16):1500-1509.

57. Emerging Risk Factors C, Erqou S, Kaptoge S, et al. Lipoprotein(a) concentration and the risk of coronary heart disease, stroke, and nonvascular mortality. JAMA. 2009;302(4):412-423.

58. Nave AH, Lange KS, Leonards $\mathrm{CO}$, et al. Lipoprotein (a) as a risk factor for ischemic stroke: a meta-analysis. Atherosclerosis. 2015;242(2):496-503.

59. Haffner S, Orchard T, Stein E, Schmidt D, LaBelle P. Effect of simvastatin on Lp(a) concentrations. Clin Cardiol. 1995;18(5):261-267.

60. Raal FJ, Giugliano RP, Sabatine MS, et al. Reduction in lipoprotein(a) with PCSK9 monoclonal antibody evolocumab (AMG 145): a pooled analysis of more than 1,300 patients in 4 phase II trials. $J$ Am Coll Cardiol. 2014;63(13):1278-1288.

61. Goldstein JL, Hobbs HH, Brown MS. Familial hypercholesterolemia. In: Scriver CR, Beaudet AL, Sly WS, Valle D, editors. The Metabolic and Molecular Bases of Inherited Disease. 8th ed. New York, NY: McGraw-Hill Inc.; 2001:2863-2913.

62. Marais AD, Raal FJ, Stein EA, et al. A dose-titration and comparative study of rosuvastatin and atorvastatin in patients with homozygous familial hypercholesterolaemia. Atherosclerosis. 2008;197(1):400-406.

63. Raal FJ, Pilcher GJ, Illingworth DR, et al. Expanded-dose simvastatin is effective in homozygous familial hypercholesterolaemia. Atherosclerosis. 1997;135(2):249-256.

64. Raal FJ, Honarpour N, Blom DJ, et al. Inhibition of PCSK9 with evolocumab in homozygous familial hypercholesterolaemia (TESLA part B): a randomised, double-blind, placebo-controlled trial. Lancet. 2015;385(9965):341-350.

65. Armitage J. The safety of statins in clinical practice. Lancet. 2007;370(9601):1781-1790.

66. Ganga HV, Slim HB, Thompson PD. A systematic review of statin-induced muscle problems in clinical trials. Am Heart J. 2014 168(1):6-15.

67. Newman CB, Tobert JA. Statin intolerance: reconciling clinical trials and clinical experience. JAMA. 2015;313(10):1011-1012.
68. Rosenson RS, Baker SK, Jacobson TA, Kopecky SL, Parker BA. The National Lipid Association's Muscle Safety Expert P. An assessment by the statin muscle safety task force: 2014 update. J Clin Lipidol. 2014;8(3 suppl):S58-S71.

69. Koren MJ, Giugliano RP, Raal FJ, et al. Efficacy and safety of longer-term administration of evolocumab (AMG 145) in patients with hypercholesterolemia: 52-week results from the open-label study of long-term evaluation against LDL-C (OSLER) randomized trial. Circulation. 2014;129(2):234-243.

70. Rojas-Fernandez CH, Goldstein LB, Levey AI, Taylor BA, Bittner V. The National Lipid Association's Safety Task F. An assessment by the statin cognitive safety task force: 2014 update. J Clin Lipidol. 2014; 8(3 suppl):S5-S16.

71. Sattar N, Preiss D, Murray HM, et al. Statins and risk of incident diabetes: a collaborative meta-analysis of randomised statin trials. Lancet 2010;375(9716):735-742.

72. Strom BL, Schinnar R, Karlawish J, Hennessy S, Teal V, Bilker WB. Statin therapy and risk of acute memory impairment. JAMA Intern Med. 2015;175(8):1399-1405.

73. Simic I, Reiner Z. Adverse effects of statins - myths and reality. Curr Pharm Des. 2015;21(9):1220-1226.

74. Kraft R, Kahn A, Medina-Franco JL, et al. A cell-based fascin bioassay identifies compounds with potential anti-metastasis or cognitionenhancing functions. Dis Model Mech. 2013;6(1):217-235.

75. Bjorkhem I, Meaney S. Brain cholesterol: long secret life behind a barrier. Arterioscler Thromb Vasc Biol. 2004;24(5):806-815.

76. Koren MJ, Blom D, Giugliano RP, et al. Safety and tolerability of very low LDL-C levels in patients treated with 52 weeks of evolocumab (AMG 145). Circulation. 2014;130:16865.

77. Hacquebard M, Carpentier YA. Vitamin E: absorption, plasma transport and cell uptake. Curr Opin Clin Nutr Metab Care. 2005;8(2):133-138.

78. Hu J, Zhang Z, Shen WJ, Azhar S. Cellular cholesterol delivery, intracellular processing and utilization for biosynthesis of steroid hormones. Nutr Metab (Lond). 2010;7:47.

79. Blom DJ, Djedjos CS, Monsalvo ML, et al. Effects of evolocumab on vitamin E and steroid hormone levels. Results from the 52-week, phase 3, double-blind, randomized, placebo-controlled DESCARTES study. Circ Res. 2015;117:731-741.

80. Qamar A, Bhatt DL. Effect of low cholesterol on steroid hormones and vitamin E levels: just a theory or real concern? Circ Res. 2015;117(8):662-664.

81. Stroes ESG, Guyton J, Farnier M, et al. Efficacy and safety of different dosing regimens of alirocumab (starting doses of $75 \mathrm{mg}$ every two weeks and $150 \mathrm{mg}$ every four weeks) versus placebo in patients with hypercholesterolemia not treated using statins: the ODYSSEY CHOICE II study. J Am Coll Cardiol. 2015;65(10S):1370.

82. Roth EM, Rader DJ, Moriarty PM, et al. A randomized phase 3 trial evaluating alirocumab every four weeks dosing as add-on to statin or as monotherapy: ODYSSEY CHOICE I. Presented at: ISA 2015, the 17th International Symposium on Atherosclerosis. May 23-26, 2015. Amsterdam: 2016. Available from: http://www.athero.org/isa2015/ ClinicalBreak/Roth.pdf. Accessed January 26, 2016.
Vascular Health and Risk Management

\section{Publish your work in this journal}

Vascular Health and Risk Management is an international, peerreviewed journal of therapeutics and risk management, focusing on concise rapid reporting of clinical studies on the processes involved in the maintenance of vascular health; the monitoring, prevention and treatment of vascular disease and its sequelae; and the involvement of

\section{Dovepress}

metabolic disorders, particularly diabetes. This journal is indexed on PubMed Central and MedLine. The manuscript management system is completely online and includes a very quick and fair peer-review system, which is all easy to use. Visit http://www.dovepress.com/ testimonials.php to read real quotes from published authors. 